\title{
Uniqueness of certain differential polynomial of $L$-functions and meromorphic functions sharing a polynomial
}

\begin{abstract}
Banerjee A. ${ }^{1}$, Bhattacharyya S. ${ }^{2}$
The purpose of this paper is to obtain some sufficient conditions to determine the relation between a meromorphic function and an $L$-function when certain differential polynomial generated by them sharing a one degree polynomial. The main theorem of the paper extends and improves all the results due to W.J. Hao, J.F. Chen [Discrete Dyn. Nat. Soc. 2018, 2018, article ID 4673165], F. Liu, X.M. Li, H.X. Yi [Proc. Japan Acad. Ser. A Math. Sci. 2017, 93 (5), 41-46], P. Sahoo, S. Halder [Tbilisi Math. J. 2018, 11 (4), 67-78].

Key words and phrases: Nevanlinna theory, $L$-function, shared value, weighted sharing, differential polynomial, uniqueness.
\end{abstract}

\footnotetext{
${ }^{1}$ Department of Mathematics, University of Kalyani, West Bengal, 741235, India

${ }^{2}$ Department of Science and Humanities, Jangipur Government Polytechnic, West Bengal, 742225, India

E-mail: abanerjee_kal@yahoo.co.in, abanerjeekal@gmail.com(Banerjee A.), saikat352@gmail.com, saikatbh89@yahoo.com (Bhattacharyya S.)
}

\section{Introduction, definitions and results}

In this paper, we use the term "L-function" to denote a Selberg class function that are Dirichlet series with the Riemann zeta function $\zeta(s)=\sum_{n=1}^{\infty} n^{-s}$ as the prototype. In the beginning of the nineteenth century R. Nevanlinna inaugurated the value distribution theory with his famous Five Value and Four Value theorems, which were the bases of uniqueness theory. Value distribution of $L$-functions concerns distribution of zeros of $L$-functions and more generally, the $c$-points of $\mathcal{L}$, that is, the zeros of the function $\mathcal{L}(s)-c$, or the values in the set of pre-images $L^{-1}=\{s \in \mathbb{C}: \mathcal{L}(s)=c\}$, where $s$ denotes complex variables and $c \in \mathbb{C} \cup\{\infty\}$. Selberg class functions are important objects in number theory. The Selberg class $S$ of $L$-functions is the set of all Dirichlet series $\mathcal{L}(s)=\sum_{n=1}^{\infty} a(n) n^{-s}$ of a complex variable $s=\sigma+$ it with $a(1)=1$, satisfying the following axioms (cf. [14,15]):

(i) Ramanujan hypothesis: $a(n) \ll n^{\varepsilon}$ for every $\varepsilon>0$;

(ii) analytic continuation: there is a nonnegative integer $k$ such that $(s-1)^{k} \mathcal{L}(s)$ is an entire function of finite order;

(iii) functional equation: $\mathcal{L}$ satisfies a functional equation of type $\Lambda_{\mathcal{L}}(s)=\omega \Lambda_{\mathcal{L}}(1-\bar{s})$, where $\Lambda_{\mathcal{L}}(s)=\mathcal{L}(s) Q^{s} \prod_{j=1}^{K} \Gamma\left(\lambda_{j} s+v_{j}\right)$ with positive real numbers $Q, \lambda_{j}$ and complex numbers $v_{j}, \omega$ with $\operatorname{Rev}_{j} \geq 0$ and $|\omega|=1$; 
(iv) Euler product hypothesis: $\mathcal{L}(s)=\prod_{p} \exp \left(\sum_{k=1}^{\infty} \frac{b\left(p^{k}\right)}{p^{k s}}\right)$ with suitable coefficients $b\left(p^{k}\right)$ satisfying $b\left(p^{k}\right) \ll p^{k \theta}$ for some $\theta<\frac{1}{2}$, where the product is taken over all prime numbers $p$.

The degree $d$ of an $L$-function $\mathcal{L}$ is defined to be

$$
d=2 \sum_{j=1}^{K} \lambda_{j}
$$

where $K$ and $\lambda_{j}$ are respectively the positive integer and the positive real number defined in axiom (iii) of the definition of $L$-function.

It should be noted, that an $L$-function can be analytically continued as meromorphic function in $\mathbb{C}$.

Throughout the paper, the term "meromorphic" will be used to mean meromorphic in the whole complex plane. For such two meromorphic functions $f, g$ and for some $a \in \mathbb{C}$, we denote by $E(a ; f)$ the collection of the zeros of $f-a$, where a zero is counted according to its multiplicity. In addition to this, when $a=\infty$, the above definition implies that we are considering the poles. In the same manner, by $\bar{E}(a ; f)$ we denote the collection of the distinct zeros or poles of $f-a$ according as $a \in \mathbb{C}$ or $a=\infty$ respectively. If $E(a ; f)=E(a ; g)$ we say that $f$ and $g$ share the value $a \mathrm{CM}$ (counting multiplicities) and if $\bar{E}(a ; f)=\bar{E}(a ; g)$, then we say that $f$ and $g$ share the value $a$ IM (ignoring multiplicities). Usually, $S(r, f)$ denotes any quantity satisfying $S(r, f)=o(T(r, f))$ for all $r$ outside of a possible exceptional set of finite linear measure.

For a meromorphic function $f$, we define the order $\rho(f)$ as

$$
\rho(f)=\limsup _{r \rightarrow \infty} \frac{\log T(r, f)}{\log r} .
$$

In 1997, I. Lahiri [5] asked the following question.

Question ([5]). What can be said about the relationship between two meromorphic functions $f$ and $g$ when two differential polynomials generated by them share some non-zero complex values?

In response to the above question plethora of investigations have been carried out on differential polynomials sharing non-zero complex values and even sets.

Recently, F. Liu, X.M. Li, H.X. Yi [11] carry forwarded the above investigations and explored over the uniqueness property of $L$-function and any meromorphic function when two differential polynomials generated by both of them share any finite complex value.

Theorem A ([11]). Let $f$ be a non-constant meromorphic function, $\mathcal{L}$ be an $L$-function and $n, k$ be two positive integers such that $n>3 k+6$. If $\left(f^{n}\right)^{(k)}-\alpha(z)$ and $\left(\mathcal{L}^{n}\right)^{(k)}-\alpha(z)$ share $(0, \infty)$, then $f=t \mathcal{L}$ for a constant $t$ satisfying $t^{n}=1$, where $\alpha(z)$ is either 1 or $z$.

In 2001, the introduction of the notion of weighted sharing [6,7], of values and sets, which is actually a scaling between CM and IM sharing, further add essence to the uniqueness literature. Below we invoke the definition.

Definition $1([6,7])$. Let $k$ be a non-negative integer or infinity. For $a \in \mathbb{C} \cup\{\infty\}$ we denote by $E_{k}(a ; f)$ the set of all a-points of $f$, where an a-point of multiplicity $m$ is counted $m$ times 
if $m \leq k$ and $k+1$ times if $m>k$. If $E_{k}(a ; f)=E_{k}(a ; g)$, we say that $f, g$ share the value $a$ with weight $k$ and denote it by $(a, k)$. The IM and CM sharing corresponds to $(a, 0)$ and $(a, \infty)$ respectively.

We also say that $f(z)$ and $g(z)$ share a polynomial $p(z)$ with weight $l$ if $f(z)-p(z)$ and $g(z)-p(z)$ share $(0, l)$.

In 2018, P. Sahoo, S. Halder [13] employed the notion of weighted sharing of values to relax the nature of sharing of value in the above theorem as follows.

Theorem B ([13]). Under the same situation as in Theorem A, if functions $\left(f^{n}\right)^{(k)}-\alpha(z)$ and $\left(\mathcal{L}^{n}\right)^{(k)}-\alpha(z)$ share $(0, l)$ and one of the following conditions is satisfied:

(i) $l \geq 2$ and $n>3 k+6$,

(ii) $l=1$ and $n>\frac{7 k}{2}+\frac{13}{2}$,

(iii) $l=0$ and $n>7 k+11$, then $f=t \mathcal{L}$ for some constant $t$ satisfying $t^{n}=1$, where $\alpha(z)$ is either 1 or $z$.

In the same year, W.J. Hao, J.F. Chen [4] generalized the differential polynomials generated by meromorphic function $f$ and $L$-function $\mathcal{L}$ to obtain a series of following four theorems.

Theorem $\mathbf{C}([4])$. Let $f$ be a non-constant meromorphic function, $\mathcal{L}$ be an $L$-function, $n, m$, $k$ be three positive integers and $\alpha, \beta$ be two constants satisfying $|\alpha|+|\beta| \neq 0$. Suppose that $\left[f^{n}\left(\alpha f^{m}+\beta\right)\right]^{(k)}$ and $\left[\mathcal{L}^{n}\left(\alpha \mathcal{L}^{m}+\beta\right)\right]^{(k)}$ share $(1, \infty)$. If $n>3 k+\tilde{m}+6$, then $f=t \mathcal{L}$, where

(i) $t$ is a constant such that $t^{n+\tilde{m}}=1$, if $\alpha \beta=0$,

(ii) $t$ is a constant such that $t^{d}=1$, if $\alpha \beta \neq 0, k \geq 2$.

Here $d=\operatorname{GCD}(n, m)$ and $\tilde{m}:=\tilde{m}(\alpha)$, where

$$
\tilde{m}(\alpha)= \begin{cases}0, & \alpha=0 \\ m, & \alpha \neq 0\end{cases}
$$

Theorem $\mathbf{D}([4])$. Let $f$ be a non-constant meromorphic function, $\mathcal{L}$ be an $L$-function and $n$, $m, k$ be three positive integers. Suppose $\left[f^{n}(f-1)^{m}\right]^{(k)}$ and $\left[\mathcal{L}^{n}(\mathcal{L}-1)^{m}\right]^{(k)}$ share $(1, \infty)$. If $n>3 k+m+6$ and $k \geq 2$, then $f \equiv \mathcal{L}$ or $f^{n}(f-1)^{m} \equiv \mathcal{L}^{n}(\mathcal{L}-1)^{m}$.

Theorem E ([4]). Under the same situation as in Theorem $C$, functions $\left[f^{n}\left(\alpha f^{m}+\beta\right)\right]^{(k)}$ and $\left[\mathcal{L}^{n}\left(\alpha \mathcal{L}^{m}+\beta\right)\right]^{(k)}$ share $(1,0)$ and $n>7 k+4 \tilde{m}+11$, then $f=t \mathcal{L}$, where

(i) $t$ is a constant such that $t^{n+\tilde{m}}=1$, if $\alpha \beta=0$,

(ii) $t$ is a constant such that $t^{d}=1$, if $\alpha \beta \neq 0, k \geq 2$.

Theorem $\mathbf{F}([4])$. Under the same situation as in Theorem $D$, if functions $\left[f^{n}(f-1)^{m}\right]^{(k)}$ and $\left[\mathcal{L}^{n}(\mathcal{L}-1)^{m}\right]^{(k)}$ share $(1,0)$ and $n>7 k+4 m+11, k \geq 2$, then $f \equiv \mathcal{L}$ or $f^{n}(f-1)^{m} \equiv$ $\mathcal{L}^{n}(\mathcal{L}-1)^{m}$

Remark 1. The differential polynomial in Theorems $D$ and $F$ becomes identical with that in Theorems $C$ and $E$ for $m=1$, so the condition $m \geq 2$ is required in Theorems $D$ and $F$.

Remark 2. Here we would like to mention that very recently X.M. Li, F. Liu, H.X. Yi [10] obtained Theorem $D$ and Theorem $F$ for $m=1$ when $n>3 k+9$ and $n>7 k+17$ respectively. Hence, the results are insignificant in context to the lower-bound of $n$ in Theorem $D$ and Theorem E. 
The purpose of the paper is to bring all the above results under a single umbrella. To this end, we consider a more generalized differential polynomial generated by a meromorphic function and an $L$-function and significantly improve all the above results.

Throughout the paper, let us denote by $P(z)$ the following $n$ degree polynomial

$$
P(z)=\sum_{j=1}^{n} a_{j} z^{j}=a_{n} \prod_{j=1}^{s}\left(z-d_{l_{j}}\right)^{l_{j}},
$$

where $a_{1}, \ldots, a_{n}(\neq 0) \in \mathbb{C}$ and $d_{l_{j}}, j=1,2, \ldots, s$, are distinct and $l_{1}, l_{2}, \ldots, l_{s}, s, n \in \mathbb{N}$ such that $\sum_{j=l}^{s} l_{j}=n$. Clearly, $P(0)=0$.

We denote by $n_{1}$ and $n_{2}$ respectively be the number of simple and multiple zeros of $P(z)$, where the zeros of $P(z)$ contributing to $n_{2}$ have been counted ignoring multiplicities.

The main result of the paper is given below. We shall show that the corollaries deduced from the main result will improve Theorems B-F by reducing the lower bound of $n$.

Throughout the paper, we will use $\eta(z)=a z+b$, where $|a|+|b| \neq 0$.

Theorem 1. Let $f$ be a non-constant meromorphic function, $\mathcal{L}$ be an $L$-function, $s$ be a nonnegative integer, $n, m, k$ be three positive integers and $\alpha, \beta$ be two constants with $|\alpha|+|\beta| \neq 0$. Suppose that $\left[P(f)\left(\alpha f^{m}+\beta\right)^{s}\right]^{(k)}-\eta(z)$ and $\left[P(\mathcal{L})\left(\alpha \mathcal{L}^{m}+\beta\right)^{s}\right]^{(k)}-\eta(z)$ share $(0, l)$. If

$$
l \geq 2 \text { and } n>\frac{k}{2}+2+2 n_{2}(k+2)+2 n_{1}+m s,
$$

or

$$
l=1 \quad \text { and } \quad n>\frac{3 k}{4}+\frac{9}{4}+\left(\frac{5 k}{2}+\frac{9}{2}\right) n_{2}+\frac{5 n_{1}}{2}+\frac{3 m s}{2}
$$

or

$$
l=0 \quad \text { and } n>2 k+\frac{7}{2}+(5 k+7) n_{2}+5 n_{1}+4 m s,
$$

then one of the following two cases holds:

(i) $\left[P(f)\left(\alpha f^{m}+\beta\right)^{s}\right]^{(k)}\left[P(\mathcal{L})\left(\alpha \mathcal{L}^{m}+\beta\right)^{s}\right]^{(k)}=\eta^{2}(z)$;

(ii) $P(f)\left(\alpha f^{m}+\beta\right)^{s}=P(\mathcal{L})\left(\alpha \mathcal{L}^{m}+\beta\right)^{s}$ or $f=t \mathcal{L}$ for a constant $t$ satisfying ${ }^{\chi_{n}}=1$, where

$$
\chi_{n}= \begin{cases}1, & \sum_{j=1}^{n-1}\left|a_{n-j}\right| \neq 0, \\ d_{1}, & a_{j}=0, \forall j=1,2, \ldots, n-1,\end{cases}
$$

$d_{1}=\operatorname{gcd}(m s+n, \ldots, m(s-i)+n, \ldots n), i=0,1, \ldots, s$.

Putting $s=0$ and $P(z)=z^{n}$ in Theorem 1, we obtain the following corollary which improves Theorem $\mathrm{B}$ by reducing the lower bound $n$.

Corollary 1. Let $f$ be a non-constant meromorphic function, $\mathcal{L}$ be an L-function and $n, k$ be two positive integers. Suppose that $\left(f^{n}\right)^{(k)}-\eta(z)$ and $\left(\mathcal{L}^{n}\right)^{(k)}-\eta(z)$ share $(0, l)$. If

$$
l \geq 2 \text { and } n>\frac{5 k}{2}+6,
$$

or

$$
l=1 \quad \text { and } n>\frac{13 k}{4}+\frac{27}{4}
$$


or

$$
l=0 \text { and } n>7 k+\frac{21}{2}
$$

then $f=t \mathcal{L}$ for a constant $t$ satisfying $t^{n}=1$.

Putting $s=1$ and $P(z)=z^{n}$ in Theorem 1 we obtain the following corollary which improves Theorem $\mathrm{C}$ and $\mathrm{E}$ by reducing the lower bound $n$.

Corollary 2. Let $f$ be a non-constant meromorphic function, $\mathcal{L}$ be an $L$-function, $n, m, k$ be three positive integers and $\alpha, \beta$ be two constants such that $|\alpha|+|\beta| \neq 0$. Suppose that $\left[f^{n}\left(\alpha f^{m}+\beta\right)\right]^{(k)}-\eta(z)$ and $\left[\mathcal{L}^{n}\left(\alpha \mathcal{L}^{m}+\beta\right)\right]^{(k)}-\eta(z)$ share $(0, l)$. If

$$
l \geq 2 \text { and } n>\frac{5 k}{2}+m+6
$$

or

$$
l=1 \quad \text { and } \quad n>\frac{13 k}{4}+\frac{3 m}{2}+\frac{27}{4}
$$

or

$$
l=0 \quad \text { and } n>7 k+4 m+\frac{21}{2},
$$

then one of the following two cases holds:

(i) when $\alpha \beta=0$, then $f=t \mathcal{L}$ for a constant $t$ satisfying $t^{n+\tilde{m}}=1$;

(ii) when $\alpha \beta \neq 0$ and $k \geq 2$, then $f \equiv t \mathcal{L}$ for a constant $t$ satisfying $t^{d}=1$.

Putting $m=1, \alpha=1, \beta=-1$ and $P(z)=z^{n}$ in Theorem 1 , we obtain the following corollary which again improves Theorem $\mathrm{D}$ and $\mathrm{F}$ by reducing the lower bound of $n$.

Corollary 3. Let $f$ be a non-constant meromorphic function, $\mathcal{L}$ be an $L$-function, $s$ be a nonnegative integer and $n, k(\geq 2)$ be two positive integers. Suppose that $\left[f^{n}(f-1)^{s}\right]^{(k)}-\eta(z)$ and $\left[\mathcal{L}^{n}(\mathcal{L}-1)^{s}\right]^{(k)}-\eta(z)$ share $(0, l)$. If

$$
l \geq 2 \text { and } n>\frac{5 k}{2}+s+6
$$

or

$$
l=1 \quad \text { and } n>\frac{13 k}{4}+\frac{3 s}{2}+\frac{27}{4}
$$

or

$$
l=0 \quad \text { and } n>7 k+4 s+\frac{21}{2}
$$

then either $f \equiv \mathcal{L}$ or $f^{n}(f-1)^{s} \equiv \mathcal{L}^{n}(\mathcal{L}-1)^{s}$.

For the standard definitions and notations of the value distribution theory we refer to [3]. But in the paper we have used some more notations and definitions which are explained below.

Definition 2 ([20]). Let $f$ and $g$ be two non-constant meromorphic functions such that $f$ and $g$ share $(a, 0)$. Let $z_{0}$ be an a-point of $f$ with multiplicity $p$, an $a$-point of $g$ with multiplicity $q$. We denote by $\bar{N}_{L}(r, a ; f)$ the reduced counting of those $a$-points of $f$ and $g$, where $p>q$, by $N_{E}^{1)}(r, a ; f)$ the counting function of those a-points of $f$ and $g$, where $p=q=1$, by $\bar{N}_{E}^{(2}(r, a ; f)$ the reduced counting function of those $a$-points of $f$ and $g$, where $p=q \geq 2$. In the same way we can define $\bar{N}_{L}(r, a ; g), N_{E}^{1)}(r, a ; g), \bar{N}_{E}^{(2}(r, a ; g)$. In a similar manner we can define $\bar{N}_{L}(r, a ; f)$ and $\bar{N}_{L}(r, a ; g)$ for $a \in \mathbb{C} \cup\{\infty\}$. 
When $f$ and $g$ share $(a, m), m \geq 1$, then $N_{E}^{1)}(r, a ; f)=N(r, a ; f \mid=1)$.

Definition $3([6,7])$. Let $f, g$ share a value $(a, 0)$. We denote by $\bar{N}_{*}(r, a ; f, g)$ the reduced counting function of those a-points of $f$ whose multiplicities differ from the multiplicities of the corresponding a-points of $g$.

Clearly, $\bar{N}_{*}(r, a ; f, g)=\bar{N}_{*}(r, a ; g, f)=\bar{N}_{L}(r, a ; f)+\bar{N}_{L}(r, a ; g)$.

Definition 4 ([8]). For $a \in \mathbb{C} \cup\{\infty\}$ and a positive integer $p$ we denote by $N(r, a ; f \mid \leq p)$ $(N(r, a ; f \mid \geq p))$ the counting function of those a-points of $f$ whose multiplicities are not greater (less) than $p$, where each a-point is counted according to its multiplicity.

$\bar{N}(r, a ; f \mid \leq p)$ and $\bar{N}(r, a ; f \mid \geq p)$ are defined similarly, where in counting the $a$-points of $f$ we ignore the multiplicities.

Also $N(r, a ; f \mid<p), N(r, a ; f \mid>p), \bar{N}(r, a ; f \mid<p)$ and $\bar{N}(r, a ; f \mid>p)$ are defined analogously.

Definition 5 ([7]). Let $p$ be a positive integer or infinity. We denote by $N_{p}(r, a ; f)$ the counting function of a-points of $f$, where an a-point of multiplicity $m$ is counted $m$ times if $m \leq p$ and $p$ times if $m>p$. Then

$$
N_{p}(r, a ; f)=\bar{N}(r, a ; f)+\bar{N}(r, a ; f \mid \geq 2)+\ldots+\bar{N}(r, a ; f \mid \geq p) .
$$

Clearly, $N_{1}(r, a ; f)=\bar{N}(r, a ; f)$.

Definition 6. Let $a$ be any value in the extended complex plane and let $k$ be an arbitrary nonnegative integer. We define

$$
\Theta(a, f)=1-\limsup _{r \rightarrow \infty} \frac{\bar{N}(r, a ; f)}{T(r, f)} \quad \text { and } \quad \delta_{k}(a, f)=1=\limsup _{r \rightarrow \infty} \frac{N_{k}(r, a ; f)}{T(r, f)} .
$$

Remark 3. From the definitions of $\Theta(a, f)$ and $\delta(a, f)$ we clearly see that

$$
0 \leq \delta_{k}(a, f) \leq \delta_{k-1}(a, f) \leq \delta_{1}(a, f) \leq \Theta(a, f) \leq 1
$$

\section{Lemmas}

Let for two non-constant meromorphic functions $F$ and $G$ we denote by $H$ the following function

$$
H=\left(\frac{F^{\prime \prime}}{F^{\prime}}-\frac{2 F^{\prime}}{F-1}\right)-\left(\frac{G^{\prime \prime}}{G^{\prime}}-\frac{2 G^{\prime}}{G-1}\right) .
$$

Lemma 1 ([17]). Suppose that $f$ is a non-constant meromorphic function and let $a_{0}, a_{1}, \ldots, a_{n}$ be finite complex numbers such that $a_{n} \neq 0$. Then

$$
T\left(r, a_{n} f^{n}+a_{n-1} f^{n-1}+\ldots+a_{1} f+a_{0}\right)=n T(r, f)+S(r, f) .
$$

Lemma 2 ([20]). Let $F, G$ be two non-constant meromorphic functions such that they share $(1,0)$ and $H \not \equiv 0$ then

$$
N_{E}^{1)}(r, 1 ; F) \leq N(r, \infty ; H)+S(r, F)+S(r, G) .
$$


Lemma 3 ([2]). Let $F, G$ be two non-constant meromorphic functions sharing $(1, l)$, where $0 \leq l<\infty$. Then

$$
\bar{N}(r, 1 ; F)+\bar{N}(r, 1 ; G)-N_{E}^{1)}(r, 1 ; F)+\left(l-\frac{1}{2}\right) \bar{N}_{*}(r, 1 ; F, G) \leq \frac{1}{2}[N(r, 1 ; F)+N(r, 1 ; G)] .
$$

Lemma 4 ([21]). Let $f$ be a non-constant meromorphic function and $k, p$ be positive integers. Then

$$
\begin{gathered}
N_{p}\left(r, 0 ; f^{(k)}\right) \leq T\left(r, f^{(k)}\right)-T(r, f)+N_{p+k}(r, 0 ; f)+S(r, f), \\
N_{p}\left(r, 0 ; f^{(k)}\right) \leq k \bar{N}(r, \infty ; f)+N_{p+k}(r, 0 ; f)+S(r, f) .
\end{gathered}
$$

Lemma 5. Let $F, G$ be two non-constant meromorphic functions such that they share $(1, l)$. Then

$$
\bar{N}_{*}(r, 1 ; F, G) \leq \frac{1}{l+1}\{\bar{N}(r, 0 ; F)+\bar{N}(r, 0 ; G)+\bar{N}(r, \infty ; F)+\bar{N}(r, \infty ; G)\}+S(r, F)+S(r, G) .
$$

Proof. The proof can be carried out in the line of the proof of [1, Lemma 2.6].

Lemma 6 ([3]). Let $f$ be a non-constant meromorphic function, $k$ be a positive integer and let $c$ be a non-zero finite complex number. Then

$$
\begin{aligned}
T(r, f) & \leq \bar{N}(r, \infty ; f)+N(r, 0 ; f)+N\left(r, c ; f^{(k)}\right)-N\left(r, 0 ; f^{(k+1)}\right)+S(r, f) \\
& \leq \bar{N}(r, \infty ; f)+N_{k+1}(r, 0 ; f)+\bar{N}\left(r, c ; f^{(k)}\right)-N_{0}\left(r, 0 ; f^{(k+1)}\right)+S(r, f)
\end{aligned}
$$

where $N_{0}\left(r, 0 ; f^{(k+1)}\right)$ is the counting function of those zeros of $f^{(k+1)}$ in $|z|<r$, which are not zeros of $f\left(f^{(k)}-c\right)$ in $|z|<r$.

Lemma 7 ([19]). Let $f$ be a non-constant meromorphic function, $\alpha(\not \equiv 0, \infty)$ be a small function of $f$. Then

$$
T(r, f) \leq \bar{N}(r, \infty ; f)+N(r, 0 ; f)+N\left(r, 0 ; f^{(k)}-\alpha\right)-N\left(r, 0 ;\left(\frac{f^{(k)}}{\alpha}\right)^{\prime}\right)+S(r, f) .
$$

Lemma 8 ([9]). Suppose that $f$ is meromorphic of finite order in the complex plane and that $f^{(k)}$ has finitely many zeros for some $k \geq 2$. Then $f$ has finitely many poles in the complex plane.

Lemma 9 ([20]). If $H \equiv 0$, then $F, G$ share $(1, \infty)$. If further $F, G$ share $(\infty, 0)$, then $F, G$ share $(\infty, \infty)$.

Lemma 10. Let $f$ and $g$ be two transcendental meromorphic functions and $H \not \equiv 0$. Let for two integers $k(\geq 1)$ and $l(\geq 0), f^{(k)}-Q, g^{(k)}-Q$ share $(0, l)$, where $Q \not \equiv 0$ is a polynomial. Then

$$
\begin{aligned}
\frac{1}{2}[T(r, f)+T(r, g)] \leq\left(\frac{k}{2}+2\right)[\bar{N}(r, \infty ; f) & +\bar{N}(r, \infty ; g)]+N_{k+2}(r, 0 ; f)+N_{k+2}(r, 0 ; g) \\
& -\left(l-\frac{3}{2}\right) \bar{N}_{*}(r, 1 ; F, G)+S(r, f)+S(r, g)
\end{aligned}
$$

where $F=\frac{f^{(k)}}{Q}$ and $G=\frac{g^{(k)}}{Q}$. 
Proof. Since $f$ and $g$ are two transcendental meromorphic functions, $F$ and $G$ are also two transcendental meromorphic functions. Let $z_{0}$ is a common simple zero of $f^{(k)}-Q$ and $g^{(k)}-Q$. Then $z_{0}$ is a common simple zero of $F-1$ and $G-1$. We can easily verify that possible pole of $H$ occur at (i) multiple zeros of $F$ and $G$, (ii) poles of $f$ and $g$, (iii) 1-points of $F$ and $G$ of different multiplicities, (iv) zeros of $F^{\prime}$ which are not the zeros of $F(F-1)$, (v) zeros of $G^{\prime}$ which are not the zeros of $G(G-1)$. Since $H$ has only simple poles, clearly we have

$$
\begin{array}{r}
N(r, \infty ; H) \leq \bar{N}(r, \infty ; f)+\bar{N}(r, \infty ; g)+\bar{N}(r, 0 ; F \mid \geq 2)+\bar{N}(r, 0 ; G \mid \geq 2)+\bar{N}_{*}(r, 1 ; F, G) \\
+\bar{N}_{\otimes}\left(r, 0 ; F^{\prime}\right)+\bar{N}_{\otimes}\left(r, 0 ; G^{\prime}\right)+O(\log r),
\end{array}
$$

where $\bar{N}_{\otimes}\left(r, 0 ; F^{\prime}\right)$ denotes the reduced counting function of those zeros of $F^{\prime}$, which are not the zeros of $F(F-1)$, and $\bar{N}_{\otimes}\left(r, 0 ; G^{\prime}\right)$ is similarly defined.

Since $f$ is a transcendental meromorphic function, we have $T(r, Q)=o\{T(r, f)\}$.

By using Lemma 7, we get

$$
\begin{aligned}
T(r, f)+T(r, g) \leq & \bar{N}(r, \infty ; f)+N(r, 0 ; f)+N(r, 1 ; F)+\bar{N}(r, \infty ; g)+N(r, 0 ; g) \\
& \quad+\bar{N}(r, 1 ; G)-N\left(r, 0 ; F^{\prime}\right)-N\left(r, 0 ; G^{\prime}\right)+S(r, f)+S(r, g) \\
\leq & \bar{N}(r, \infty ; f)+N_{k+1}(r, 0 ; f)+\bar{N}(r, 1 ; F)+\bar{N}(r, \infty ; g)+N_{k+1}(r, 0 ; g) \\
& \quad+\bar{N}(r, 1 ; G)-N_{0}\left(r, 0 ; F^{\prime}\right)-N_{0}\left(r, 0 ; G^{\prime}\right)+S(r, f)+S(r, g),
\end{aligned}
$$

where $N_{0}\left(r, 0 ; F^{\prime}\right)$ is the counting function of those zeros of $F^{\prime}$ in $|z|<r$, which are not the zeros of $f(F-1)$ in $|z|<r$.

Now using Lemma 1, 2, 3 and (5), we get

$$
\begin{aligned}
\bar{N}(r, 1 ; F)+\bar{N}(r, 1 ; G) \leq & \frac{1}{2}[N(r, 1 ; F)+N(r, 1 ; G)]+N_{E}^{1)}(r, 1 ; F)-\left(l-\frac{1}{2}\right) \bar{N}_{*}(r, 1 ; F, G) \\
\leq & \frac{1}{2}\{T(r, f)+T(r, g)\}+\left(\frac{k}{2}+1\right) \bar{N}(r, \infty ; f)+\left(\frac{k}{2}+1\right) \bar{N}(r, \infty ; g) \\
& +\bar{N}(r, 0 ; F \mid \geq 2)+\bar{N}(r, 0 ; G \mid \geq 2)-\left(l-\frac{3}{2}\right) \bar{N}_{*}(r, 1 ; F, G) \\
& +\bar{N}_{\otimes}\left(r, 0 ; F^{\prime}\right)+\bar{N}_{\otimes}\left(r, 0 ; G^{\prime}\right)+O(\log r) .
\end{aligned}
$$

So from (6) and (7), we obtain

$$
\begin{aligned}
\frac{1}{2}[T(r, f)+T(r, g)] \leq( & \left.\frac{k}{2}+2\right)[\bar{N}(r, \infty ; f)+\bar{N}(r, \infty ; g)]+N_{k+1}(r, 0 ; f)+\bar{N}(r, 0 ; F \mid \geq 2) \\
& +N_{k+1}(r, 0 ; g)+\bar{N}(r, 0 ; G \mid \geq 2)-\left(l-\frac{3}{2}\right) \bar{N}_{*}(r, 1 ; F, G)+\bar{N}_{\otimes}\left(r, 0 ; F^{\prime}\right) \\
& +\bar{N}_{\otimes}\left(r, 0 ; G^{\prime}\right)-N_{0}\left(r, 0 ; F^{\prime}\right)-N_{0}\left(r, 0 ; G^{\prime}\right)+S(r, f)+S(r, g) \\
\leq & \left(\frac{k}{2}+2\right)[\bar{N}(r, \infty ; f)+\bar{N}(r, \infty ; g)]+N_{k+1}(r, 0 ; f)+\bar{N}(r, 0 ; f \mid \geq k+2) \\
& +\bar{N}(r, 0 ; F|\geq 2| f \neq 0)+N_{k+1}(r, 0 ; g)+\bar{N}(r, 0 ; g \mid \geq k+2) \\
& +\bar{N}(r, 0 ; G|\geq 2| g \neq 0)-\left(l-\frac{3}{2}\right) \bar{N}_{*}(r, 1 ; F, G)+\bar{N}_{\otimes}\left(r, 0 ; F^{\prime}\right) \\
& +\bar{N}_{\otimes}\left(r, 0 ; G^{\prime}\right)-N_{0}\left(r, 0 ; F^{\prime}\right)-N_{0}\left(r, 0 ; G^{\prime}\right)+S(r, f)+S(r, g) \\
\leq( & \left.\frac{k}{2}+2\right)[\bar{N}(r, \infty ; f)+\bar{N}(r, \infty ; g)]+N_{k+2}(r, 0 ; f)+N_{k+2}(r, 0 ; g) \\
& -\left(l-\frac{3}{2}\right) \bar{N}_{*}(r, 1 ; F, G)+S(r, f)+S(r, g) .
\end{aligned}
$$


Lemma 11. Let $f, g$ be two transcendental meromorphic functions and $F, G$ be defined as in Lemma 10. Then either $f^{(k)} g^{(k)} \equiv Q^{2}$ or $f \equiv g$, whenever $f$ and $g$ satisfies one of the following conditions:

(i) $l \geq 2$ and $\left(\frac{k}{2}+2\right)\{\Theta(\infty, f)+\Theta(\infty, g)\}+\delta_{k+2}(0, f)+\delta_{k+2}(0, g)>k+5$;

(ii) $l=1$ and $\left(\frac{3 k}{4}+\frac{9}{4}\right)\{\Theta(\infty, f)+\Theta(\infty, g)\}+\delta_{k+2}(0, f)+\delta_{k+2}(0, g)$

$$
+\frac{1}{4}\left\{\delta_{k+1}(0, f)+\delta_{k+1}(0, g)\right\}>\frac{3 k}{2}+6
$$

(iii) $l=0$ and $\left(2 k+\frac{7}{2}\right)\{\Theta(\infty, f)+\Theta(\infty, g)\}+\delta_{k+2}(0, f)+\delta_{k+2}(0, g)$

$$
+\frac{3}{2}\left\{\delta_{k+1}(0, f)+\delta_{k+1}(0, g)\right\}>4 k+11 \text {. }
$$

Proof. Case 1. Let $H \not \equiv 0$. We consider the following cases.

Subcase 1.1. Let $l \geq 2$. Then from Lemma 10, we get

$$
\begin{aligned}
\frac{1}{2}[T(r, f)+T(r, g)] \leq & \left(\frac{k}{2}+2\right)[\bar{N}(r, \infty ; f)+\bar{N}(r, \infty ; g)] \\
& +N_{k+2}(r, 0 ; f)+N_{k+2}(r, 0 ; g)+S(r, f)+S(r, g) \\
\leq & \left.\left(\frac{k}{2}+3\right)-\left(\frac{k}{2}+2\right) \Theta(\infty, f)-\delta_{k+2}(0, f)\right] T(r, f) \\
& \quad+\left[\left(\frac{k}{2}+3\right)-\left(\frac{k}{2}+2\right) \Theta(\infty, g)-\delta_{k+2}(0, g)\right] T(r, g)+S(r, f)+S(r, g),
\end{aligned}
$$

i.e.,

$$
\begin{aligned}
{\left[\left(\frac{k}{2}+2\right) \Theta(\infty, f)\right.} & \left.+\delta_{k+2}(0, f)-\left(\frac{k}{2}+\frac{5}{2}\right)\right] T(r, f) \\
& +\left[\left(\frac{k}{2}+2\right) \Theta(\infty, g)+\delta_{k+2}(0, g)-\left(\frac{k}{2}+\frac{5}{2}\right)\right] T(r, g) \leq S(r, f)+S(r, g) .
\end{aligned}
$$

Without loss of generality, we may suppose that there exists a set $I$ with infinite linear measure such that $T(r, g) \leq T(r, f), r \in I$. Then for $r \in I$, we have

$$
\left[\left(\frac{k}{2}+2\right)\{\Theta(\infty, f)+\Theta(\infty, g)\}+\delta_{k+2}(0, f)+\delta_{k+2}(0, g)-(k+5)\right] T(r, f) \leq S(r, f),
$$

which contradicts (8).

Subcase 1.2. Let $l=1$. So from Lemma, 4,5 and 10 , we have

$$
\begin{aligned}
\frac{1}{2}[T(r, f)+T(r, g)] \leq & \left(\frac{k}{2}+2\right)[\bar{N}(r, \infty ; f)+\bar{N}(r, \infty ; g)] \\
& +N_{k+2}(r, 0 ; f)+N_{k+2}(r, 0 ; g)+\frac{1}{2} \bar{N}_{*}(r, 1 ; F, G)+S(r, f)+S(r, g) \\
\leq & \left(\frac{k}{2}+\frac{9}{4}\right)[\bar{N}(r, \infty ; f)+\bar{N}(r, \infty ; g)]+N_{k+2}(r, 0 ; f) \\
& \quad+N_{k+2}(r, 0 ; g)+\frac{1}{4}\{\bar{N}(r, 0 ; F)+\bar{N}(r, 0 ; G)\}+S(r, f)+S(r, g) \\
\leq & \left(\frac{3 k}{4}+\frac{9}{4}\right)[\bar{N}(r, \infty ; f)+\bar{N}(r, \infty ; g)]+N_{k+2}(r, 0 ; f)+N_{k+2}(r, 0 ; g) \\
& \quad+\frac{1}{4}\left\{N_{k+1}(r, 0 ; f)+N_{k+1}(r, 0 ; g)\right\}+S(r, f)+S(r, g) \\
\leq & {\left[\left(\frac{3 k}{4}+\frac{7}{2}\right)-\left(\frac{3 k}{4}+\frac{9}{4}\right) \Theta(\infty, f)-\delta_{k+2}(0, f)-\frac{1}{4} \delta_{k+1}(0, f)\right] T(r, f) } \\
+ & {\left[\left(\frac{3 k}{4}+\frac{7}{2}\right)-\left(\frac{3 k}{4}+\frac{9}{4}\right) \Theta(\infty, g)-\delta_{k+2}(0, g)-\frac{1}{4} \delta_{k+1}(0, g)\right] T(r, g) } \\
& \quad+S(r, f)+S(r, g),
\end{aligned}
$$


i.e.

$$
\begin{aligned}
& {\left[\left(\frac{3 k}{4}+\frac{9}{4}\right) \Theta(\infty, f)+\delta_{k+2}(0, f)+\frac{1}{4} \delta_{k+1}(0, f)-\left(\frac{3 k}{4}+3\right)\right] T(r, f)} \\
& \quad+\left[\left(\frac{3 k}{4}+\frac{9}{4}\right) \Theta(\infty, g)+\delta_{k+2}(0, g)+\frac{1}{4} \delta_{k+1}(0, g)-\left(\frac{3 k}{4}+3\right)\right] T(r, g) \leq S(r, f)+S(r, g) .
\end{aligned}
$$

Without loss of generality, we may suppose that there exists a set $I$ with infinite linear measure such that $T(r, g) \leq T(r, f), r \in I$. Then for $r \in I$, we have

$$
\begin{aligned}
{\left[\left(\frac{3 k}{4}+\frac{9}{4}\right)\{\Theta(\infty, f)+\Theta(\infty, g)\}\right.} & +\delta_{k+2}(0, f)+\delta_{k+2}(0, g) \\
& \left.+\frac{1}{4}\left\{\delta_{k+1}(0, f)+\delta_{k+1}(0, g)\right\}-\left(\frac{3 k}{2}+6\right)\right] T(r, f) \leq S(r, f),
\end{aligned}
$$

which contradicts (9).

Subcase 1.3. Let $l=0$. So from Lemmas 4,5 and 10 , we have

$$
\begin{aligned}
\frac{1}{2}[T(r, f)+T(r, g)] \leq & \left.\frac{k}{2}+2\right)[\bar{N}(r, \infty ; f)+\bar{N}(r, \infty ; g)] \\
& +N_{k+2}(r, 0 ; f)+N_{k+2}(r, 0 ; g)+\frac{3}{2} \bar{N}_{*}(r, 1 ; F, G)+S(r, f)+S(r, g) \\
\leq & \left(\frac{k}{2}+\frac{7}{2}\right)[\bar{N}(r, \infty ; f)+\bar{N}(r, \infty ; g)]+N_{k+2}(r, 0 ; f)+N_{k+2}(r, 0 ; g) \\
& \quad+\frac{3}{2}\{\bar{N}(r, 0 ; F)+\bar{N}(r, 0 ; G)\}+S(r, f)+S(r, g) \\
\leq & \left(2 k+\frac{7}{2}\right)[\bar{N}(r, \infty ; f)+\bar{N}(r, \infty ; g)]+N_{k+2}(r, 0 ; f)+N_{k+2}(r, 0 ; g) \\
& \quad+\frac{3}{2}\left\{N_{k+1}(r, 0 ; f)+N_{k+1}(r, 0 ; g)\right\}+S(r, f)+S(r, g) \\
\leq & \left.(2 k+6)-\left(2 k+\frac{7}{2}\right) \Theta(\infty, f)-\delta_{k+2}(0, f)-\frac{3}{2} \delta_{k+1}(0, f)\right] T(r, f) \\
& \quad+\left[(2 k+6)-\left(2 k+\frac{7}{2}\right) \Theta(\infty, g)-\delta_{k+2}(0, g)-\frac{3}{2} \delta_{k+1}(0, g)\right] T(r, g) \\
\leq & S(r, f)+S(r, g),
\end{aligned}
$$

i.e.,

$$
\begin{aligned}
{[(2 k} & \left.\left.+\frac{7}{2}\right) \Theta(\infty, f)+\delta_{k+2}(0, f)+\frac{3}{2} \delta_{k+1}(0, f)-\left(2 k+\frac{11}{2}\right)\right] T(r, f) \\
& +\left[\left(2 k+\frac{7}{2}\right) \Theta(\infty, g)+\delta_{k+2}(0, g)+\frac{3}{2} \delta_{k+1}(0, g)-\left(2 k+\frac{11}{2}\right)\right] T(r, g) \leq S(r, f)+S(r, g) .
\end{aligned}
$$

Without loss of generality, we may suppose that there exists a set $I$ with infinite linear measure such that $T(r, g) \leq T(r, f), r \in I$. Then for $r \in I$, we have

$$
\begin{aligned}
{\left[\left(2 k+\frac{7}{2}\right)\{\Theta(\infty, f)\right.} & +\Theta(\infty, g)\}+\delta_{k+2}(0, f)+\delta_{k+2}(0, g) \\
& \left.+\frac{3}{2}\left\{\delta_{k+1}(0, f)+\delta_{k+1}(0, g)\right\}-(4 k+11)\right] T(r, f) \leq S(r, f),
\end{aligned}
$$

which contradicts (10). 
Case 2. Let $H \equiv 0$. On integration we get from (4)

$$
F \equiv \frac{A G+B}{C G+D}
$$

where $A, B, C, D$ are complex constants satisfying $A D-B C \neq 0$. Also by Mokhon'ko's Lemma (see [12])

$$
T(r, f)=T(r, g)+S(r, f) .
$$

From Lemma 9 we see that $F, G$ share $(1, \infty)$, which again implies $F, G$ share $(1,2)$. So we consider only the inequality (8) and so from (12) the condition becomes

$$
\left(\frac{k}{2}+2\right)\{\Theta(\infty, f)+\Theta(\infty, g)\}+\delta_{k+2}(0, f)+\delta_{k+2}(0, g)>k+5 .
$$

As $A D-B C \neq 0$, so both $A$ and $C$ cannot be simultaneously zero. Thus we consider the following cases.

Subcase 2.1. Suppose $A C \neq 0$. Then $F-\frac{A}{C}=\frac{B C-A D}{C(C G+D)} \neq 0$. So $F$ omits the value $\frac{A}{C}$. Now by using Lemma 6, we get

$$
\begin{aligned}
T(r, f) & \leq N_{k+1}(r, 0 ; f)+\bar{N}(r, \infty ; f)+\bar{N}\left(r, \frac{A}{C} ; F\right)-N_{0}\left(r, 0 ; F^{\prime}\right)+S(r, f) \\
& \leq N_{k+2}(r, 0 ; f)+\bar{N}(r, \infty ; f)+S(r, f),
\end{aligned}
$$

which yields $\delta_{k+2}(0, f)+\Theta(\infty ; f) \leq 1$.

Thus from (13), we get

$$
\left(\frac{k}{2}+1\right) \Theta(\infty, f)+\left(\frac{k}{2}+2\right) \Theta(\infty, g)+\delta_{k+2}(0, g)>k+4
$$

which is a contradiction from the Definition 6.

Subcase 2.2. Suppose $A C=0$.

Subcase 2.2.1. Let $A=0$ and $C \neq 0$. Then (11) becomes $F=\frac{1}{\gamma G+\delta}$, where $\gamma=\frac{C}{D}$ and $\delta=\frac{D}{C}$.

If $F$ has no 1-point, i.e. 1 is a Picard Exceptional value, then by using Lemma 6, we get

$$
\begin{aligned}
T(r, f) & \leq N_{k+1}(r, 0 ; f)+\bar{N}(r, \infty ; f)+\bar{N}(r, 1 ; F)-N_{0}\left(r, 0 ; F^{\prime}\right)+S(r, f) \\
& \leq N_{k+2}(r, 0 ; f)+\bar{N}(r, \infty ; f)+S(r, f),
\end{aligned}
$$

which again yields $\delta_{k+2}(0, f)+\Theta(\infty ; f) \leq 1$, and similarly as above we arrive at a contradiction.

So let $F$ has some 1-point. Then $\gamma+\delta=1$. Since $C \neq 0$, so $\gamma \neq 0$ and thus

$$
F=\frac{1}{\gamma G+1-\gamma}
$$

By using Lemma 6, we get

$$
\begin{aligned}
T(r, f) & \leq N_{k+1}(r, 0 ; f)+\bar{N}(r, \infty ; f)+\bar{N}\left(r, \frac{1}{1-\gamma} ; F\right)-N_{0}\left(r, 0 ; F^{\prime}\right)+S(r, f) \\
& \leq N_{k+1}(r, 0 ; f)+\bar{N}(r, \infty ; f)+\bar{N}(r, 0 ; G)+S(r, f)+S(r, g) \\
& \leq N_{k+1}(r, 0 ; f)+N_{k+1}(r, 0 ; g)+\bar{N}(r, \infty ; f)+k \bar{N}(r, \infty ; g)+S(r, f)+S(r, g) \\
& \leq N_{k+2}(r, 0 ; f)+N_{k+2}(r, 0 ; g)+\bar{N}(r, \infty ; f)+k \bar{N}(r, \infty ; g)+S(r, f)+S(r, g),
\end{aligned}
$$


which yields by using (12), $\delta_{k+2}(0, f)+\delta_{k+2}(0, g)+\Theta(\infty, f)+k \Theta(\infty, g) \leq k+2$. Thus from (13) we get

$$
\left(\frac{k}{2}+1\right) \Theta(\infty, f)+\left(2-\frac{k}{2}\right) \Theta(\infty, g)>3,
$$

which is a contradiction from the Definition 6.

Thus $\gamma=1$ and $F G \equiv 1$, i.e. $f^{(k)} g^{(k)} \equiv Q^{2}$.

Subcase 2.2.2. Let $A \neq 0$ and $C=0$. Then $D \neq 0$ and (11) becomes $F=\lambda G+\mu$, where $\lambda=\frac{A}{D}$ and $\mu=\frac{B}{D}$.

If $F$ has no 1-point then the case can be treated similarly as done above.

So let $F$ has some 1 -point. Then $\lambda+\mu=1$ such that $\mu \neq 0$. If $\lambda \neq 1$ then by using Lemma 6 , we get

$$
\begin{aligned}
T(r, f) & \leq N_{k+1}(r, 0 ; f)+\bar{N}(r, 1-\lambda ; F)+\bar{N}(r, \infty ; f)-N_{0}\left(r, 0 ; F^{\prime}\right)+S(r, f) \\
& \leq N_{k+1}(r, 0 ; f)+\bar{N}(r, 0 ; G)+\bar{N}(r, \infty ; f)+S(r, f) \\
& \leq N_{k+1}(r, 0 ; f)+N_{k+1}(r, 0 ; g)+k \bar{N}(r, \infty ; g)+\bar{N}(r, \infty ; f)+S(r, f) \\
& \leq N_{k+2}(r, 0 ; f)+N_{k+2}(r, 0 ; g)+k \bar{N}(r, \infty ; g)+\bar{N}(r, \infty ; f)+S(r, f),
\end{aligned}
$$

which by using (12) again yields $\delta_{k+2}(0, f)+\delta_{k+2}(0, g)+\Theta(\infty, f)+k \Theta(\infty, g) \leq k+2$, and similarly as above we arrive at a contradiction.

Thus $\lambda=1$ and so $F \equiv G$, which can be rewritten as

$$
f=g+Q_{1}
$$

where $Q_{1}$ is a polynomial of degree $\gamma_{Q_{1}} \leq k-1$. Combining (14) and Nevanlinna's three small functions theorem (see [18, Theorem 1.36]) we get

$$
\begin{aligned}
T(r, g) & \leq \bar{N}(r, \infty ; g)+\bar{N}(r, 0 ; g)+\bar{N}\left(r, 0 ; g+Q_{1}\right)+S(r, g) \\
& =\bar{N}(r, \infty ; g)+\bar{N}(r, 0 ; g)+\bar{N}(r, 0 ; f)+S(r, g) .
\end{aligned}
$$

Again form (14) we get $T(r, f)=T(r, g)+O(\log r)$. From this and (15) we get

$$
\Theta(0, f)+\Theta(0, g)+\Theta(\infty, g) \leq 2
$$

From (13), (16) and Remark 3, we get

$$
\left(\frac{k}{2}+2\right) \Theta(\infty, f)+\left(\frac{k}{2}+1\right) \Theta(\infty, g)>k+3
$$

Hence from (17) and Remark 3 we get a contradiction. Thus $Q_{1}=0$ and so we get from (14) that $f=g$. This completes the proof.

\section{Proof of the Theorems}

Proof of Theorem 1. Let $d$ be the degree of the $L$-function $\mathcal{L}$. Therefore, by Steuding [15, p. 150] we have

$$
T(r, \mathcal{L})=\frac{d}{\pi} r \log r+O(r)
$$


We set the functions $F_{1}$ and $G_{1}$ as follows

$$
F_{1}=\frac{F^{(k)}}{\eta(z)}, \quad G_{1}=\frac{G^{(k)}}{\eta(z)},
$$

where $F=P(f)\left(\alpha f^{m}+\beta\right)^{s}$ and $G=P(\mathcal{L})\left(\alpha \mathcal{L}^{m}+\beta\right)^{s}$.

Clearly, since $F^{(k)}-\eta(z), G^{(k)}-\eta(z)$ share $(0, l)$, hence $F_{1}, G_{1}$ share $(1, l)$.

Noting that an $L$-function has at most one pole $z=1$ in the complex plane, we deduce by Lemmas 1 and 7 and Valiron-Mokhonko's lemma (cf. [12]) that

$$
\begin{aligned}
(n+m s) T(r, \mathcal{L})+S(r, f) & =T(r, G) \\
& \leq \bar{N}(r, \infty ; G)+N(r, 0 ; G)+\bar{N}\left(r, 1 ; G_{1}\right)-N\left(r, 0 ; G_{1}^{\prime}\right)+S(r, f) \\
& \leq \bar{N}(r, \infty ; G)+N_{k+1}(r, 0 ; G)+\bar{N}\left(r, 1 ; G_{1}\right)-N_{0}\left(r, 0 ; G_{1}^{\prime}\right)+S(r, f) \\
& \leq \bar{N}(r, \infty ; \mathcal{L})+(k+1) \bar{N}(r, 0 ; G)+\bar{N}\left(r, 1 ; G_{1}\right)+S(r, f) \\
& \leq(k+1)(n+m s) T(r, \mathcal{L})+\bar{N}\left(r, 1 ; F_{1}\right)+S(r, f),
\end{aligned}
$$

where $N_{0}\left(r, 0 ; G_{1}^{\prime}\right)$ is the counting function of those zeros of $G_{1}^{\prime}$ in $|z|<r$, which are not the zeros of $G$ and $G_{1}-1$ in $|z|<r$. This implies

$$
-k(n+m s) T(r, \mathcal{L}) \leq T\left(r, F^{(k)}\right)+S(r, f) .
$$

By (18) we see that $\mathcal{L}$ is a transcendental meromorphic function. Combining this with (20), [18, Theorem 1.5] and the assumption of the lower bound of $n$, we deduce that $F^{(k)}$ and so $f$ is a transcendental meromorphic function.

Now we set

$$
\begin{aligned}
\Delta_{1}=\left(\frac{k}{2}+2\right)\{\Theta(\infty, F)+\Theta(\infty, G)\} & +\delta_{k+2}(0, F)+\delta_{k+2}(0, G) \\
\Delta_{2}=\left(\frac{3 k}{4}+\frac{9}{4}\right)\{\Theta(\infty, F)+\Theta(\infty, G)\} & +\delta_{k+2}(0, F)+\delta_{k+2}(0, G) \\
& +\frac{1}{4}\left\{\delta_{k+1}(0, F)+\delta_{k+1}(0, G)\right\}
\end{aligned}
$$

and

$$
\begin{aligned}
\Delta_{3}=\left(2 k+\frac{7}{2}\right)\{\Theta(\infty, F)+\Theta(\infty, G)\} & +\delta_{k+2}(0, F)+\delta_{k+2}(0, G) \\
& +\frac{3}{2}\left\{\delta_{k+1}(0, F)+\delta_{k+1}(0, G)\right\} .
\end{aligned}
$$

Using Lemma 1, we have

$$
\begin{aligned}
\Theta(\infty, F) & =1-\limsup _{r \rightarrow \infty} \frac{\bar{N}(r, \infty ; F)}{T(r, F)} \\
& =1-\limsup _{r \rightarrow \infty} \frac{\bar{N}(r, \infty ; f)}{(n+m s) T(r, f)+O(1)} \geq 1-\frac{1}{n+m s}, \\
\delta_{k+2}(0, F) & =1-\limsup _{r \rightarrow \infty} \frac{N_{k+2}(r, 0 ; F)}{T(r, F)} \\
& \geq 1-\limsup _{r \rightarrow \infty} \frac{N_{k+2}(r, 0 ; P(f))+N_{k+2}\left(r, 0 ;\left(\alpha f^{m}+\beta\right)^{s}\right)}{(n+m s) T(r, f)+O(1)} \\
& \geq 1-\frac{n_{2}(k+2)+n_{1}+m s}{n+m s} .
\end{aligned}
$$


Similarly,

$$
\begin{gathered}
\delta_{k+2}(0, G) \geq 1-\frac{n_{2}(k+2)+n_{1}+m s}{n+m s}, \\
\delta_{k+1}(0, F) \geq 1-\frac{n_{2}(k+1)+n_{1}+m s}{n+m s}, \quad \delta_{k+1}(0, G) \geq 1-\frac{n_{2}(k+1)+n_{1}+m s}{n+m s} .
\end{gathered}
$$

Since an $L$-function has at most one pole at $z=1$ in the complex plane, we have

$$
N(r, \mathcal{L}) \leq \log r+O(1)
$$

So using (18) we deduce that

$$
\Theta(\infty, G)=1
$$

Case 1. Let $l \geq 2$. By using (21), (24)-(26) and (27), we have

$$
\Delta_{1} \geq(k+6)-\frac{\left(\frac{k}{2}+2\right)+2 n_{2}(k+2)+2 n_{1}+2 m s}{n+m s} .
$$

By (28) and the assumption $n>\left(\frac{k}{2}+2\right)+2 n_{2}(k+2)+2 n_{1}+m s$, we have $\Delta_{1}>k+5$. Thus by Lemma 11 we get either $F^{(z)} G^{(k)}=\eta^{2}(z)$ or $F \equiv G$.

Let $F \equiv G$, i.e.

$$
P(f)\left(\alpha f^{m}+\beta\right)^{s}=P(\mathcal{L})\left(\alpha \mathcal{L}^{m}+\beta\right)^{s} .
$$

Now we set

$$
H=\frac{f}{\mathcal{L}} .
$$

If $H$ is a non-constant meromorphic function, then we get (29).

Suppose $H$ is a constant. Then from (30), we get

$$
\begin{aligned}
& {\left[a_{n} f^{n}+a_{n-1} f^{n-1}+\ldots+a_{1} z\right]\left[\left(\alpha f^{m}\right)^{s}+\left(\begin{array}{l}
s \\
1
\end{array}\right)\left(\alpha f^{m}\right)^{s-1} \beta+\ldots+\left(\begin{array}{l}
s \\
s
\end{array}\right) \beta^{s}\right]} \\
& \quad=\left(a_{n} \mathcal{L}^{n}+a_{n-1} \mathcal{L}^{n-1}+\ldots+a_{1} z\right)\left[\left(\alpha \mathcal{L}^{m}\right)^{s}+\left(\begin{array}{l}
s \\
1
\end{array}\right)\left(\alpha \mathcal{L}^{m}\right)^{s-1} \beta+\ldots+\left(\begin{array}{l}
s \\
s
\end{array}\right) \beta^{s}\right],
\end{aligned}
$$

i.e.

$$
\begin{aligned}
\sum_{i=0}^{s}\left(\begin{array}{l}
s \\
i
\end{array}\right) \beta^{i}\left[a_{n} \mathcal{L}^{m(s-i)+n}\left(H^{m(s-i)+n}-1\right)\right. & +a_{n-1} \mathcal{L}^{m(s-i)+n-1}\left(H^{m(s-i)+n-1}-1\right)+\ldots \\
& \left.+a_{1} \mathcal{L}^{m(s-i)+1}\left(H^{m(s-i)+1}-1\right)\right]=0
\end{aligned}
$$

which implies $H^{\chi_{n}}=1$, where

$$
\chi_{n}= \begin{cases}1, & \sum_{j=1}^{n-1}\left|a_{n-j}\right| \neq 0, \\ d_{1}, & a_{j}=0, \forall j=1,2, \ldots, n-1,\end{cases}
$$


$d_{1}=\operatorname{gcd}(m s+n, \ldots, m(s-i)+n, \ldots n), i=0,1, \ldots, s$. Therefore, $f=t \mathcal{L}$ for a constant $t$ satisfying $t^{\chi_{n}}=1$.

Case 2. Let $l=1$. By using (22), (24)-(27), we have

$$
\Delta_{2} \geq \frac{3 k}{2}+7-\frac{\frac{3 k}{4}+\frac{9}{4}+\left(\frac{5 k}{2}+\frac{9}{2}\right) n_{2}+\frac{5 n_{1}}{2}+\frac{5 m s}{2}}{n+m s} .
$$

By (31) and the assumption $n>\frac{3 k}{4}+\frac{9}{4}+\left(\frac{5 k}{2}+\frac{9}{2}\right) n_{2}+\frac{5 n_{1}}{2}+\frac{3 m s}{2}$, we have $\Delta_{2}>\frac{3 k}{2}+6$. Thus by Lemma 11 we get either $F^{(z)} G^{(k)}=\eta^{2}(z)$ or $F \equiv G$. Proceeding in the same manner as done in Case 1, we get the conclusion.

Case 3. Let $l=0$. By using (23), (24)-(27), we have

$$
\Delta_{3} \geq 4 k+12-\frac{2 k+\frac{7}{2}+(5 k+7) n_{2}+5 n_{1}+5 m s}{n+m s} .
$$

By (32) and the assumption $n>2 k+\frac{7}{2}+(5 k+7) n_{2}+5 n_{1}+4 m s$, we have $\Delta_{3}>4 k+11$. Thus by Lemma 11 we get either $F^{(z)} G^{(k)}=\eta^{2}(z)$ or $F \equiv G$. Proceeding in the same manner as done in Case 1, we get the conclusion.

Proof of Corollary 2. We set the functions $F_{1}$ and $G_{1}$ as follows.

$$
F_{1}=\frac{F^{(k)}}{\eta(z)}, \quad G_{1}=\frac{G^{(k)}}{\eta(z)},
$$

where $F=f^{n}\left(\alpha f^{m}+\beta\right)$ and $G=\mathcal{L}^{n}\left(\alpha \mathcal{L}^{m}+\beta\right)$. Clearly, since $F^{(k)}-\eta(z), G^{(k)}-\eta(z)$ share $(0, l)$, hence $F_{1}, G_{1}$ share $(1, l)$.

Then using the same procedure as adopted in Theorem 1, we obtain either $F^{(z)} G^{(k)}=\eta^{2}(z)$ or $F \equiv G$.

Subcase 1.1. Suppose $F^{(k)} G^{(k)}=\eta^{2}(z)$, i.e.

$$
\left\{f^{n}\left(\alpha f^{m}+\beta\right)\right\}^{(k)}\left\{\mathcal{L}^{n}\left(\alpha \mathcal{L}^{m}+\beta\right)\right\}^{(k)}=\eta^{2}(z) .
$$

Subcase 1.1.1. Let $\alpha \beta \neq 0$. Then using (18), (33), Lemma 1 and a result from Whittaker [16, p.82] and the definition of the order of a meromorphic function we have

$$
\begin{aligned}
\rho(f)=\rho\left(\left(f^{n}\left(\alpha f^{m}+\beta\right)\right)^{(k)}\right) & =\rho\left(\frac{\left(f^{n}\left(\alpha f^{m}+\beta\right)\right)^{(k)}}{\eta^{2}(z)}\right) \\
& =\rho\left(\left(\mathcal{L}^{n}\left(\alpha \mathcal{L}^{m}+\beta\right)\right)^{(k)}\right)=\rho\left(\mathcal{L}^{n}\left(\alpha \mathcal{L}^{m}+\beta\right)\right)=\rho(\mathcal{L})=1 .
\end{aligned}
$$

By (34) we can see that $f$ is a transcendental meromorphic function. Since an $L$-function has at most one pole at $z=1$ in the complex plane, we deduce by (33) that $\frac{\left(f^{n}\left(\alpha f^{m}+\beta\right)\right)^{(k)}}{\eta^{2}(z)}$ has at most one zero at $z=1$ in the complex plane. Now, as $\eta(z)$ is a polynomial, so the zero comes only from $\left(f^{n}\left(\alpha f^{m}+\beta\right)\right)^{(k)}$. Combining this with (34), Lemma 8 and the assumption $k \geq 2$, we obtain that $f^{n}\left(\alpha f^{m}+\beta\right)$ has finitely many poles and so $f$ has finitely many poles in the complex plane. This together with (33) implies that $\frac{\left(\mathcal{L}^{n}\left(\alpha \mathcal{L}^{m}+\beta\right)\right)^{(k)}}{\eta^{2}(z)}$ and so $\left(\mathcal{L}^{n}\left(\alpha \mathcal{L}^{m}+\beta\right)\right)^{(k)}$ has at most finitely many zeros in the complex plane. Moreover, by the assumptions (1)-(3), we deduce that $\mathcal{L}$ has at most finitely many zeros. Thus,

$$
\mathcal{L}=R_{1} e^{A_{1} z+B_{1}},
$$


where $R_{1}$ is a rational function, $A_{1} \neq 0$ and $B_{1}$ are constants. By (35) and Hayman [3, p.7] we have

$$
T(r, \mathcal{L})=T\left(r, R_{1} e^{A_{1} z+B_{1}}\right) \leq \frac{\left|A_{1}\right| r}{\pi}(1+o(1))+O(\log r),
$$

which contradicts (18).

Subcase 1.1.2. Let $\alpha \beta=0$. As $|\alpha|+|\beta| \neq 0$, we have to consider the following two cases.

Subcase 1.1.2.1 Let $\alpha \neq 0, \beta=0$. Then (33) becomes $\left\{f^{n+m} \alpha^{m}\right\}^{(k)}\left\{\mathcal{L}^{n+m} \alpha^{m}\right\}^{(k)}=\eta^{2}(z)$. Let $z_{0}$ be a zero of $\mathcal{L}$ of order $\lambda$. Then we can get that $z_{0}$ is a pole of $f$ of order $\chi$, satisfying $(n+m) \lambda-k=(n+m) \chi+k$, i.e. $(n+m)(\lambda-\chi)=2 k$, which implies $n+m \leq 2 k$, contradicting the assumptions (1)-(3). Hence, $\mathcal{L}$ has no zeros and so $\mathcal{L}=R_{2} e^{A_{2} z+B_{2}}$, where $R_{2}$ is a rational function and $A_{2}(\neq 0), B_{2}$ are constants. Thus, adopting the same procedure as in Subcase 1.1.1 we arrive at a contradiction.

Subcase 1.1.2.2 Let $\alpha=0, \beta \neq 0$. Then (33) becomes $\left\{f^{n} \beta^{m}\right\}^{(k)}\left\{\mathcal{L}^{n} \beta^{m}\right\}^{(k)}=\eta^{2}(z)$. By using the argument as in Subcase 1.1.2.1, we obtain $n(\lambda-\chi)=2 k$, which again contradicts the assumptions (1)-(3). Thus, in the similar way we arrive at a contradiction as in Subcase 1.1.2.1.

Subcase 1.2. Let $F \equiv G$, i.e.

$$
f^{n}\left(\alpha f^{m}+\beta\right)=\mathcal{L}^{n}\left(\alpha \mathcal{L}^{m}+\beta\right) .
$$

So $f$ and $\mathcal{L}$ share $(\infty, \infty)$.

Subcase 1.2.1. Let $\alpha \beta \neq 0$. Taking $H=\frac{f}{\mathcal{L}}$, we get

$$
\alpha \mathcal{L}^{n+m}\left(H^{n+m}-1\right)=-\beta \mathcal{L}^{n}\left(H^{n}-1\right) .
$$

Suppose $H$ is a non-constant meromorphic function. Then by (37) we have

$$
\frac{\alpha \mathcal{L}^{m}}{\beta}=-\frac{H^{n}-1}{H^{n+m}-1}
$$

Let $d=\operatorname{gcd}(n, m)$. Then clearly $H^{d}=1$ is the common factor of $H^{n}-1$ and $H^{n+m}-1$. Therefore, (38) can be rewritten as

$$
\frac{\alpha \mathcal{L}^{m}}{\beta}=-\frac{1+H+\ldots+H^{n-d}}{1+H+\ldots+H^{n+m-d}}
$$

By (39) and Lemma 1 we have

$$
T(r, \mathcal{L})=T\left(r, \frac{1+H+\ldots+H^{n-d}}{1+H+\ldots+H^{n+m-d}}\right)=(n+m-d) T(r, H)+O(1) .
$$

Also

$$
\begin{aligned}
\rho(f)=\rho\left(f^{n}\left(\alpha f^{m}+\beta\right)\right)=\rho\left(\left(f^{n}\left(\alpha f^{m}+\beta\right)\right)^{(k)}\right) & =\rho\left(\left(\mathcal{L}^{n}\left(\alpha \mathcal{L}^{m}+\beta\right)\right)^{(k)}\right) \\
& =\rho\left(\mathcal{L}^{n}\left(\alpha \mathcal{L}^{m}+\beta\right)\right)=\rho(\mathcal{L})=1 .
\end{aligned}
$$

By (37), (40), (41) and by the second fundamental theorem we have

$$
\bar{N}(r, \infty ; \mathcal{L})=\sum_{j=1}^{n} \bar{N}\left(r, \gamma_{j} ; H\right)+o(T(r, H)) \geq(n+m-d-1) T(r, H),
$$


as $r \rightarrow \infty$. Here $\lambda_{1}, \lambda_{2}, \ldots, \lambda_{n+m-d}$ are $n+m-d$ distinct finite complex numbers satisfying $\lambda_{j} \neq 1$ and $\lambda_{j}^{n+m-d}=1$ for $1 \leq j \leq n+m-d$. Noting that $\mathcal{L}$ is a transcendental meromorphic function such that $\mathcal{L}$ has at most one pole $z=1$ in the complex plane, we deduce by (42) that there exists some small positive number $\varepsilon$ satisfying $0<\varepsilon<1$, such that

$$
(n+m-d-1-\varepsilon) T(r, H) \leq \bar{N}(r, \infty ; \mathcal{L})=\log r+O(1) .
$$

By (43) and the assumptions (1)-(3) and $k \geq 2$ we deduce that $H$ is a non-constant rational function.

Since $f$ and $\mathcal{L}$ share $(\infty, \infty)$, it follows from the construction of $H$, that the poles of $H$ only comes from the zeros of $\mathcal{L}$ and so they are finite in number. As a result $\mathcal{L}$ has a representation

$$
\mathcal{L}=R_{3} e^{A_{3} z+B_{3}},
$$

where $R_{3}$ is a rational function and $A_{3}(\neq 0), B_{3}$ are constants. Thus proceeding the similar way as adopted in Subcase 1.1.1 we arrive at a contradiction.

When $H$ is a constant meromorphic function then from (36) we get

$$
\alpha \mathcal{L}^{n+m}\left(H^{n+m}-1\right)+\beta \mathcal{L}^{n}\left(H^{n}-1\right)=0,
$$

which implies $H^{d}=1$. Therefore, $f=t \mathcal{L}$ for a constant $t$ satisfying $t^{d}=1$.

Subcase 1.2.2. Let $\alpha \beta=0$. As $|\alpha|+|\beta| \neq 0$, so we have to consider the following two cases.

Subcase 1.2.2.1. Let $\alpha=0$ and $\beta \neq 0$. Then clearly we get $f=t \mathcal{L}$, where $t$ is a constant satisfying $t^{n}=1$.

Subcase 1.2.2.2. Let $\alpha \neq 0$ and $\beta=0$. Then clearly we see that $f=t \mathcal{L}$, where $t$ is a constant satisfying $t^{n+m}=1$.

\section{References}

[1] Banerjee A., Bhattacharyya S. On the uniqueness of meromorphic functions and its difference operator sharing values or sets. Rend. Circ. Mat. Palermo (2) 2018, 67 (1), 75-85. doi:10.1007/s12215-016-0295-1

[2] Banerjee A. Uniqueness of meromorphic functions sharing two sets with finite weight II. Tamkang J. Math. 2010, 41 (4), 379-392. doi:10.5556/j.tkjm.41.2010.787

[3] Hayman W.K. Meromorphic Functions. The Clarendon Press, Oxford, 1964.

[4] Hao W.J., Chen J.F. Uniqueness of L-functions concerning certain differential polynomials. Discrete Dyn. Nat. Soc. 2018, 2018, article ID 4673165. doi:10.1155/2018/4673165

[5] Lahiri I. Uniqueness of meromorphic functions as governed by their differential polynomials. Yokohama Math. J. 1997, 44 (2), 147-156.

[6] Lahiri I. Weighted sharing and uniqueness of meromorphic functions. Nagoya Math. J. 2001, 161, $193-206$. doi:10.1017/S0027763000027215

[7] Lahiri I. Weighted value sharing and uniqueness of meromorphic functions. Complex Var. Elliptic Equ. 2001,46 (3), 241-253. doi:10.1080/17476930108815411

[8] Lahiri I. Value distribution of certain differential polynomials. Int. J. Math. Math. Sci. 2001,28 (2), $83-91$. doi:10.1155/S0161171201011036

[9] Langley J.K. The second derivative of a meromorphic function of finite order. Bull. Lond. Math. Soc. 2003, 35 (1), 97-108. doi:10.1112/S0024609302001558 
[10] Li X.M., Liu F., Yi H.X. Results on L-functions of certain differential polynomial sharing one finite value. Filomat 2019, 33 (18), 5767-5776. doi:10.2298/FIL1918767L

[11] Liu F., Li X.M., Yi H.X. Value distribution of L-functions concerning shared values and certain differential polynomials. Proc. Japan Acad. Ser. A Math. Sci. 2017, 93 (5), 41-46. doi:10.3792/pjaa.93.41

[12] Mokhon'ko A.Z. On Nevanlinna characteristics of certain meromorphic functions. Theory of funct. funct. anal. appl. 1971, 14, 83-87. (in Russian)

[13] Sahoo P., Halder S. Uniqueness results related to L-functions and certain differential polynomials. Tbilisi Math. J. 2018, $11(4), 67-78$.

[14] Selberg A. Old and new conjectures and results about a class of Dirichlet series. In: Proc. of the Amal Conference on Analytic Number Theory, Maiori, Salerno, Italy, 1989, Univ. Salerno, Salerno, 1992.

[15] Steuding J. Value-distribution of L-functions. In: Morel J.-M., Teissier B. (Eds.) Lecture Notes in Mathematics. Springer, Berlin, 2007.

[16] Whittaker J.M. The order of the derivative of a meromorphic function. J. Lond. Math. Soc. 1936, s1-11 (2), 82-87. doi:10.1112/jlms/s1-11.2.82

[17] Yang C.C. On deficiencies of differential polynomials II. Math. Z. 1972, 125, 107-112.

[18] Yang C.C., Yi H.X. Uniqueness Theory of Meromorphic functions. Kluwer Academic Publishers, Dordrecht, 2003.

[19] Yang L. Normality for families of meromorphic functions. Sci. Sinica Ser. A. 1986, 29 (12), 1263-1274.

[20] Yi H.X. Meromorphic functions that share one or two values II. Kodai Math. J. 1999, 22 (2), $264-272$.

[21] Zhang J.L., Yang L.Z. Some results related to a conjecture of R. Bruck. J. Inequal. Pure Appl. Math. 2007, 8 (1), art. 18.

Банірджі А., Бхаттачарія C. Єдиність деяких диференціальних поліномів L-функиій та мероморфних функцій, щзо розділяють поліном // Карпатські матем. публ. - 2021. — Т.13, №1. - С. 189-206.

Метою цієї статті є отримання деяких достатніх умов для визначення зв'язку між мероморфною функцією і $L$-функцією, коли певний диференціальний поліном, породжений ними, розділяє поліном першого степеня. Основна теорема статті розширює та вдосконалює всі результати наступних авторів: W.J. Hao, J.F. Chen [Discrete Dyn. Nat. Soc. 2018, 2018, article ID 4673165], F. Liu, X.M. Li, H.X. Yi [Proc. Japan Acad. Ser. A Math. Sci. 2017, 93 (5), 41-46], P. Sahoo, S. Halder [Tbilisi Math. J. 2018, 11 (4), 67-78].

Ключові слова і фррази: теорія Неванлінни, L-функція, спільні значення, зважена спільність, диференціальний поліном, єАиність. 